\title{
Article
}

\section{Evaluation of the Scandium Affinity under the Iron Coexistence Using Radiation Grafted Adsorbents}

\author{
Natsuki HAYASHI $^{1 *}$, Hiroyuki HOSHINA ${ }^{2}$, Haruyo AMADA ${ }^{2}$, Takeshi YAMANOBE ${ }^{1}$ \\ and Noriaki SEKO ${ }^{1,2}$ \\ ${ }^{I}$ Division of Molecular Science, Faculty of Science and Technology, Gunma University, Japan \\ ${ }^{2}$ Environmetal Polymer Group, Quantum Beam Science Center, Sector of Nuclear Science Research, Japan Atomic \\ Energy Agency, Japan
}

(Manuscript received May 30, 2014; Accepted August 11, 2014)

\begin{abstract}
Fibrous phosphoric acid adsorbents for scandium were synthesized using two methods; by radiationinduced graft polymerization of glycidyl methacrylate (GMA) with subsequent chemical modification (GMA-gP), and by direct radiation grafting with phosphoric monomer having both vinyl and phosphoric groups (HMP-g) onto a polypropylene coated by polyethylene nonwoven fabric. The resulting grafted fibrous adsorbent, HMP-g, having the grafted long side chain, has high affinity for scandium ( $\mathrm{Sc}(\mathrm{III})$ ) adsorption even under the coexistence with Fe(III) ion. The breakthrough capacity for $0.5 \mathrm{ppm} \mathrm{Sc(III)}$ solution with Fe(III) interference was $10.9 \mu$ mol-Sc(III)/g-adsorbent for GMA-gP, and $17.8 \mu \mathrm{mol}-$ $\mathrm{Sc}(\mathrm{III}) / \mathrm{g}$-adsorbent for HMP-g, respectively.
\end{abstract}

Keywords: Radiation Grafting, Scandium, Phosphoric adsorbent

\section{Introduction}

Rare metals are essential to modern life and are indispensable to industrial development in Japan. In particular, scandium, Sc, which is a kind of rare-earth element, is widely applied as an industrial key material in products such as battery separator or metal halide lamp ${ }^{1)}$. Currently, most of the rareearth elements are imported. From the view point of stockpiling, Japanese focused on recovering these elements from water streams, such as hot springs ${ }^{2}$, and from urban mines which also contain different kinds of rare-earth metals. If we can successfully collect these metals, it will contribute to the security of metal resource supply. In order to collect such metals from urban mines, strong acids are commonly used to elute them in aqueous media. However, other heavy metals which are non-valuable such as ferric ion, Fe(III), are also eluted at the same time. It is desirable to establish a system for enhancing scandium uptake from hot spring waters which contain iron as interfering element or to separate and purify neodymium or dysprosium from scandium and iron contaminated waters. Hence, it is important to develop an adsorbent with high resistance towards the acidic eluting solution and with high affinity for the target metal ion.

In order to overcome these demands, a fibrous phosphoric adsorbent was synthesized by radiation graft polymerization. The adsorbents prepared by this method are superior to those prepared in another way because new functions can be imparted to conventional polymer materials while maintaining its original properties. Specifically, hydrophilic trunk materials can be applied because the target ion is in aqueous media and the shape of material can be changed which prevents the deterioration of adsorption performance from increase in water pressure. Nonwoven fabric, a kind of fibrous material used as trunk polymer, provides rapid treatment performance of aqueous media due to high void fraction ${ }^{3,4)}$. Irradiation of

* Corresponding author. Fax.: 81273469381

E-mail: seko.noriaki@jaea.go.jp (N. Seko). 
nonwoven fabrics with electron beam creates active sites which can initiate graft polymerization when exposed to the monomer solution. This method is advantageous compared to other techniques because it can be performed at room temperature and without using chemical catalyst ${ }^{5}$.

The objective of this work is to synthesize two types of adsorbents containing phosphoric functional group, which has good affinity for $\mathrm{Sc}(\mathrm{III})$, by radiation graft polymerization to establish an environment friendly method for recovery of rareearth metals, and to store the metals for the future supply.

\section{Experimental \\ 2.1 Preparation of Grafted Adsorbents 2.1.1 Preparation of GMA-gP}

Polypropylene coated polyethylene nonwoven fabric (NF) obtained from Kurashiki Textile Manufacturing Co., Ltd. was used as trunk material for radiation graft polymerization. The NF with $0.1 \mathrm{~mm}$ apparent thickness was cut into $5 \times 8 \mathrm{~cm}$ and sealed in polyethylene bag, purged with nitrogen gas, and irradiated with electron beam of $2 \mathrm{MeV}$ energy and $3 \mathrm{~mA}$ current at dry ice temperature. The irradiated NF was put into a glass ampoule and the air inside the ampoule was immediately evacuated by a vacuum pump. In this work, we synthesized two types of phosphoric adsorbents. For the first one, the NF was irradiated to a total dose of $20 \mathrm{kGy}$ and grafted in 5\% glycidyl methacrylate (GMA) aqueous emulsion composed of $94.5 \%$ distilled water and $0.5 \%$ Tween 20 for $1 \mathrm{~h}$ at $40{ }^{\circ} \mathrm{C}^{6)}$. After grafting, the GMA-grafted NF was rinsed with methanol and distilled water to remove residual GMA monomer and homopolymer. After drying the GMA-grafted NF under reduced pressure, the amount of GMA grafted on trunk NF was calculated and expressed as degree of grafting (Dg). Dg was defined by the following equation:

$$
\operatorname{Dg}[\%]=100\left[\left(W_{1}-W_{0}\right) / W_{0}\right]
$$

where $W_{0}$ is weight of NF before grafting and $W_{1}$ that after grafting.

The GMA-grafted NF was reacted with $98 \%$ phosphoric acid for $2 \mathrm{~h}$ at $80^{\circ} \mathrm{C}$ to introduce phosphoric group by ring opening reaction with the epoxy unit on the GMA-grafted NF. The phosphorylated of GMA-grafted NF is hereinafter referred to as GMA-gP adsorbent.

\subsubsection{Preparation of HMP-g}

The other type of adsorbent was prepared as follows. The NF irradiated to $20 \mathrm{kGy}$ was grafted for $3 \mathrm{~h}$ at $60{ }^{\circ} \mathrm{C}$ in phosphoric monomer aqueous solution which has contains vinyl bonds and pendant phosphoric group in the monomer (HMP) composed of 2-hydroxyl methacrylic phosphoric acid di-ester (50\%) and mono-ester (50\%) as shown in Fig. 1. It is well known that an ester compound undergoes hydrolysis in acidic medium. In this
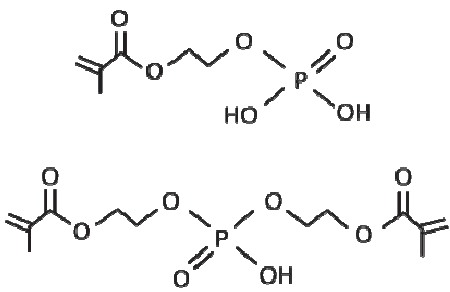

Fig.1 Structure of phosphoric monomers.

experiment, HMP having both mono- and di-ester structure was selected to prevent the ester hydrolysis group ${ }^{7}$. By the introduction of mono-ester and di-ester, its acid resistance would be increased due to forming a network structure among intermolecular. The ester bond does not be decomposed under the acid condition, therefore. The HMP monomer solution was prepared by dilution with distilled water to make a $20 \mathrm{wt} \%$ solution. After grafting, the HMP-grafted NF (HMP-g) was rinsed similar to the GMA-gP treatment, and the Dg value was obtained from equation (1). Each monomer solution was emulsified with homogenizer and purged with $\mathrm{N}_{2}$ gas ${ }^{8)}$.

\subsection{Adsorption Tests}

The adsorption performances of both grafted phosphoric adsorbents in acidic media were evaluated by batch and column mode adsorption. In the batch adsorption test, $0.01 \mathrm{~g}$ adsorbent was put into a $50 \mathrm{ml}$ of $0.5 \mathrm{ppm} \mathrm{Sc(III)} \mathrm{solution} \mathrm{which} \mathrm{also}$ contains $0.5 \mathrm{ppm} \mathrm{Fe}(\mathrm{III})$ ion. The $\mathrm{pH}$ of solution was adjusted by adding nitric acid to mimic the urban mine solution, and the most suitable $\mathrm{pH}$ condition in acidic media to adsorb of $\mathrm{Sc}$ (III) was determined. After mixing in the metal solution for $2 \mathrm{~h}$, the adsorbents were removed from the solution and the remaining metal concentration was measured by inductively coupled plasma atomic emission spectrometry (Perkin Elmer Co., Ltd., ICP-AES). Adsorption performance was expressed by the following equations;

$$
\text { Adsorption }[\%]=100\left[\left(A_{1}-A_{0}\right) / A_{0}\right]
$$

\section{Distribution coefficient \\ $=($ mg-metal per g-adsorbent $) /($ mg-metal per $g$-solution $)$}

where $A_{0}$ and $A_{1}$ are the metal concentrations before and after batch adsorption, respectively.

To evaluate the adsorption capacity, a solution containing $0.5 \mathrm{ppm}$ of each metal was pumped into a column with $7 \mathrm{~mm}$ inner diameter and packed with either 5 pieces of GMA-gP or 10 pieces of HMP-g. The total adsorbent volume was adjusted to $0.12 \mathrm{~cm}^{3}$. The column mode adsorption of $\mathrm{Sc}(\mathrm{III})$ at $\mathrm{pH} 2.9$, which has the highest performance of $\mathrm{pH}$ dependency, were tested under the coexistence with $\mathrm{Fe}(\mathrm{III})$ ion at a space velocity (SV) $250 \mathrm{~h}^{-1}$. The $\mathrm{SV}$ was calculated by dividing the flow rate of solution by the adsorbent volume in the column. The outflow 
solution was fractionally collected by a fraction collector. The breakthrough point was defined when the concentration at the outlet reached $5 \%$ of that at the inlet. The breakthrough capacity of the adsorbent for metal was calculated as the following equation;

$$
\begin{aligned}
& \text { Breakthrough capacity }[\mathrm{mol} / \mathrm{kg}] \\
& =\int_{0}^{V_{0.05}}\left(C_{0}-C\right) d V / W_{g}
\end{aligned}
$$

where $C_{0}, C, V$ and $V_{0.05}$ are the metal concentrations of the feed and effluent, effluent volume and effluent volume when $\mathrm{C} / \mathrm{C}_{0}$ reaches 0.05 , respectively. $W g$ is the weight of the phosphoric adsorbent.

\subsection{Characterization of Phosphoric Adsorbent}

The grafted NFs were characterized by using FTIR spectrometer (Perkin Elmer Co., Ltd., Spectrum One) with sapphire attenuated total reflectance (ATR) attachment.

The thermogravimetric analyses were performed using a TGA analyzer (SEIKO Instruments Inc., TG-TDA 6200). Thermogravimetric measurements were performed under dry state and nitrogen atmosphere with $10{ }^{\circ} \mathrm{C} / \mathrm{min}$ heating rate.

The water content of the both phosphoric materials was determined as the following equation;

$$
\text { Water content }[\%]=100\left[\left(W_{w}-W_{d}\right) / W_{d}\right]
$$

where $W_{w}$ and $W_{d}$ are the weight of each phosphoric adsorbent in the wet and dry condition, respectively.

\section{Result and Discussion}

\subsection{Fibrous Phosphoric Grafted Adsorbents}

The conditions of grafting, chemical modification and resulting Dg were listed in Table1. In the graft polymerization, both Dg's increased monotonously with grafting reaction time. The Dg's of the GMA-gP and HMP-g were $267 \%$ and $194 \%$ at reaction times of $1 \mathrm{~h}$ and $3 \mathrm{~h}$, respectively. The epoxy groups were modified to phosphoric groups by reaction with phosphoric acid. The functional group density of the GMA-gP was $2.1 \mathrm{mmol} / \mathrm{g}$-adsorbent while that of HMP-g was 1.2 $\mathrm{mmol} / \mathrm{g}$-adsorbent. The evidences for introduction of phosphoric group for GMA-gP and HMP-g was shown by IR spectroscopy. Fig. 2 shows the FTIR spectra of trunk NF, GMA-gP and HMP-g. The trunk NF and the two adsorbents have a common absorption band around $3000 \mathrm{~cm}^{-1}$. It is probably due to vibration of $\mathrm{C}-\mathrm{H}$ bond in the trunk NF. The peaks at 1730,1250 and $1150 \mathrm{~cm}^{-1}$ were corresponding to $\mathrm{C}=\mathrm{O}$, $\mathrm{P}=\mathrm{O}$ and $\mathrm{O}-\mathrm{P}-\mathrm{O}$ bands appeared for both adsorbents ${ }^{7)}$.

\subsection{Batch Adsorption of Sc(III) and Fe(III)}

The effect of $\mathrm{pH}$ on $\mathrm{Sc}(\mathrm{III})$ adsorption in the acidic solution was studied by batch mode adsorption. The HMP-g adsorbent
Table 1 Grafting conditions for phosphoric adsorbents

\begin{tabular}{ccc}
\hline & GMA-gP $^{6)}$ & HMP-g \\
\hline Concentration & GMA 5\% & HMP 20\% \\
Solvent & $\begin{array}{c}\text { Tween20 0.5\% } \\
\text { distilled water } 94.5 \%\end{array}$ & distilled water 80\% \\
Grafting Time & 1 hour & 3 hours \\
Grafting Temp. & $40{ }^{\circ} \mathrm{C}$ & $60{ }^{\circ} \mathrm{C}$ \\
Degree of Grafting & $267 \%$ & $194 \%$ \\
\hline
\end{tabular}

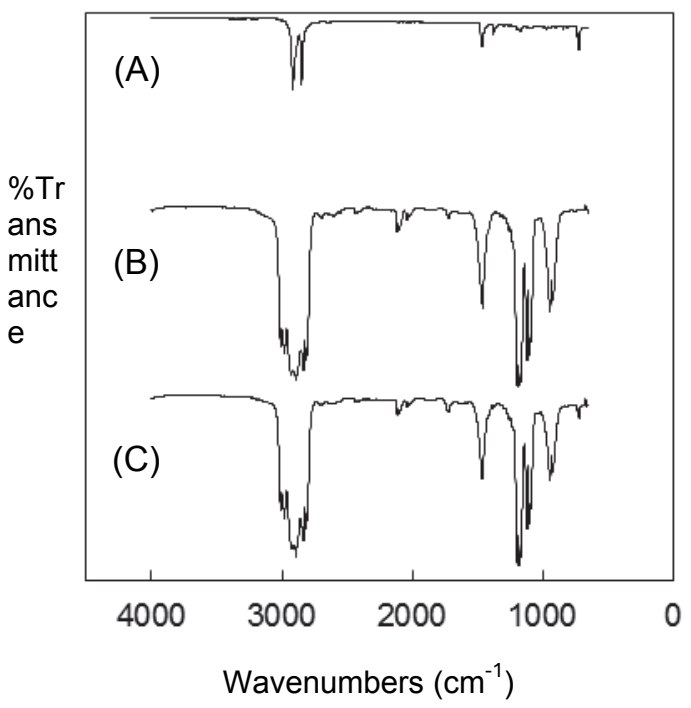

Fig.2 FTIR spectra of (A) trunk material, (B) GMA-gP, (C) HMP-g.

was mixed in a $\mathrm{Sc}(\mathrm{III}) / \mathrm{Fe}$ (III) solution with $0.5 \mathrm{ppm}$ concentration from $\mathrm{pH} 1$ to 3 . Almost $90 \%$ of $\mathrm{Sc}(\mathrm{III})$ was removed by the adsorbent after $2 \mathrm{~h}$. The selectivity of the adsorbent was evaluated by calculating the distribution coefficient given by equation (3).

Fig. 3 shows the effects of $\mathrm{pH}$ on the distribution coefficients for $\mathrm{Sc}$ (III) and $\mathrm{Fe}$ (III) of both phosphoric adsorbents. The highest distribution coefficient was attained at $\mathrm{pH}$ 3. Although the distribution coefficient values for GMA-gP were almost similar with very minimal differences, the values for HMP-g showed significant differences on the $\mathrm{pH}$ range studied. In the individual adsorption test that was carried out for each metal, the distribution coefficient value of HMP-g were $6.9 \times 10^{4}$ for $\mathrm{Sc}(\mathrm{III})$ and $3.4 \times 10^{3}$ for $\mathrm{Fe}(\mathrm{III})$, respectively. This result indicated that HMP-g adsorbent also has almost similar affinities towards $\mathrm{Sc}(\mathrm{III})$ and $\mathrm{Fe}(\mathrm{III})$. However, when $\mathrm{Sc}(\mathrm{III})$ and $\mathrm{Fe}(\mathrm{III})$ are both present in the solution, the obtained distribution coefficients were $1.9 \times 10^{6}$ and $2.2 \times 10^{4}$ for $\mathrm{Sc}$ (III) and $\mathrm{Fe}(\mathrm{III})$, respectively. The water contents of GMA-gP and HMP-g were $58 \%$ and $102 \%$, respectively, showing almost 2 times difference. This indicated that the hydrophilicity of 
HMP-g is higher than that of GMA-gP, then a surface diffusion on grafted side chain was also faster. This result implied that the grafted side chain of HMP-g could be longer than GMA-gP one. As a result, HMP-g adsorbent had higher affinity for Sc(III) than GMA-gP adsorbent.

\subsection{Column Mode Adsorption}

The column mode adsorption tests were performed by supplying a feed solution containing $0.5 \mathrm{ppm}$ of $\mathrm{Sc}(\mathrm{III})$ and $\mathrm{Fe}(\mathrm{III})$ with pH 2.9 through an adsorbent packed column with continuous flow at SV $250 \mathrm{~h}^{-1}$. The result of the column mode adsorption tests were shown in Fig. 4 as breakthrough curves. Breakthrough curve is the relation of bed volume (BV) and $\mathrm{C} / \mathrm{C}_{0}$ which is the concentration ratio of the $\mathrm{Sc}$ (III) and $\mathrm{Fe}(\mathrm{III})$ solution before $\left(\mathrm{C}_{0}\right)$ and after (C) passing the column respectively. BV's at breakthrough point were 422 on $\mathrm{Sc}$ (III) and 524 on Fe(III) for GMA-gP and 655 on Sc(III) and 547 on Fe(III) for HMP-g. The calculated breakthrough capacities were $10.9 \mu \mathrm{mol}-\mathrm{Sc}(\mathrm{III}) / \mathrm{g}$-adsorbent and $10.2 \mu \mathrm{mol}-\mathrm{Fe}(\mathrm{III}) / \mathrm{g}$ adsorbent for GMA-gP, while for HMP-g, the breakthrough capacities were $17.8 \mu \mathrm{mol}-\mathrm{Sc}(\mathrm{III}) / \mathrm{g}$-adsorbent, $10.2 \mu \mathrm{mol}$ $\mathrm{Fe}(\mathrm{III}) / \mathrm{g}$-adsorbent. From this result, the adsorption rate of HMP-g was higher than that of GMA-gP. In addition, the adsorption saturated capacities of HMP-g were $90.0 \mu \mathrm{mol}-$ $\mathrm{Sc}(\mathrm{III}) / \mathrm{g}$-adsorbent, $\quad 51.0 \quad \mu \mathrm{mol}-\mathrm{Fe}(\mathrm{III}) / \mathrm{g}$-adsorbent, respectively. Although both adsorbents have phosphoric groups, the selectivity of HMP-g for $\mathrm{Sc}(\mathrm{III})$ has improved in comparison to GMA-gP. In the case of HMP-g, it has di-ester group in monomer so the resulting grafted adsorbent has become a network structure suitable for $\mathrm{Sc}$ (III) adsorption. The high durability under acidic solutions could be deduced from the TGA analysis. Degradation steps of the grafted materials normally have two steps, with the first stage corresponding to the degradation of the grafted side chain. The defference of degradation temperature between the two phosphoric adsorbents was $11^{\circ} \mathrm{C}$. Since the heat stability of HMP-g is higher than that of GMA-gP one, the adsorption performances in acidic solution could be expected.

\section{References}

1) T. Yoshida, Recycling of precious and rare metals, NTS, (2007) (in Japanese).

2) N. Seko, H. Hoshina, N. Kasai, Y. Ueki, M. Tamada, T. Kiryu, K. Tanaka and M. Takahashi, J. Ion Exchange, 21, 117-122 (2010).

3) N. Kubota, J. Membrane Sci., 22 (5), 271-277 (1997)

4) T. Shinohara, T. Fukuoka, T. Katoh and T. Mukaiyama, Basic knowledge for nonwoven fabric, Nikkan Kogyo Shinbun, (2012) (in Japanese).

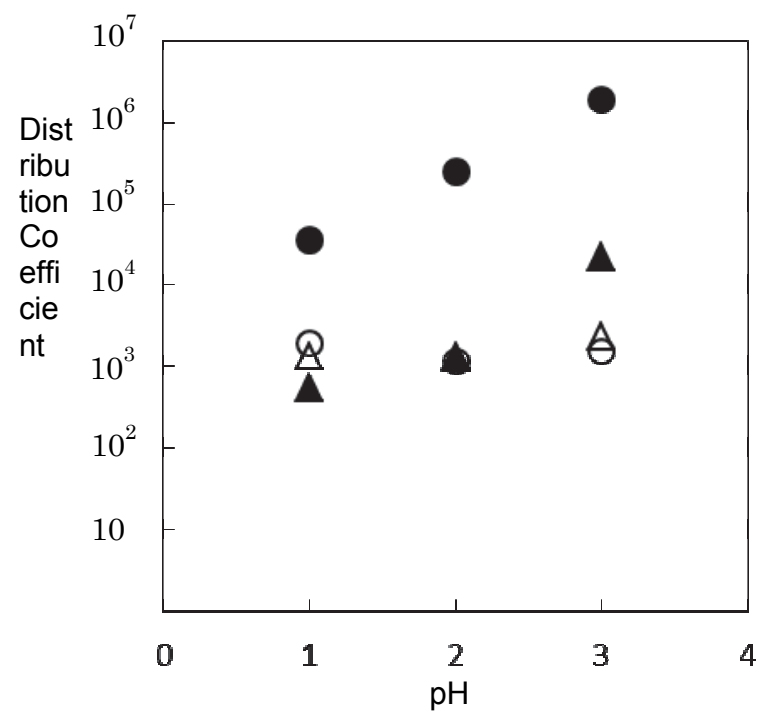

Fig. 3 Effect of pH on distribution coefficient for HMP-g adsorbent in $0.5 \mathrm{ppm}$ Sc(III) solution under coexistence with $\mathrm{Fe}(\mathrm{III})(\bullet: \mathrm{Sc}(\mathrm{III}), \Delta: \mathrm{Fe}(\mathrm{III}))$ and for GMA-gP adsorbent in $0.5 \mathrm{ppm}$ Sc(III) solution under coexistence with $\mathrm{Fe}$ (III) $(\mathrm{O}: \mathrm{Sc}(\mathrm{III}), \triangle: \mathrm{Fe}(\mathrm{III}))$.

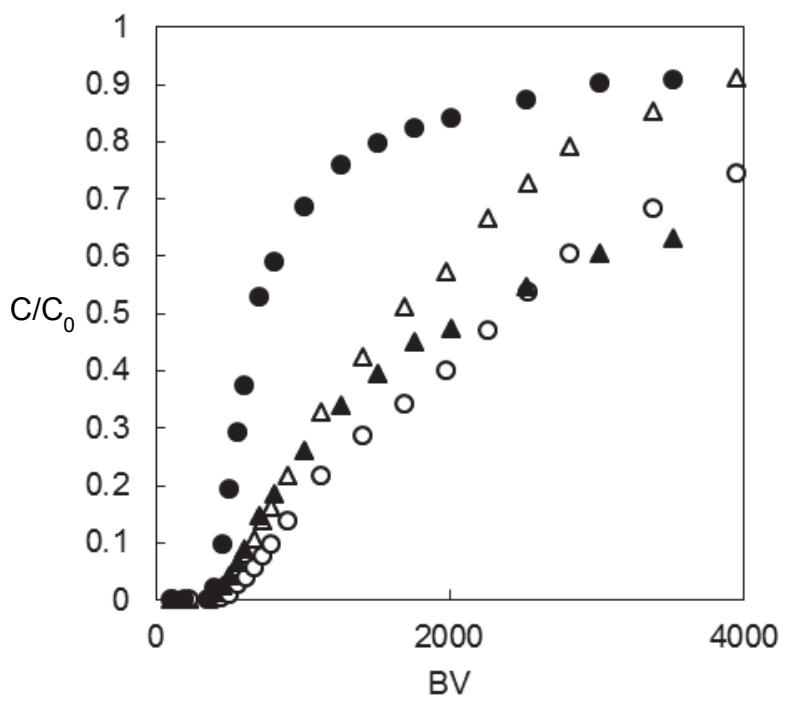

Fig.4 The adsorptive behavior of GMA-gP ( $\bullet: \mathrm{Sc}(\mathrm{III}), \Delta: \mathrm{Fe}(\mathrm{III}))$ and HMP-g (०: Sc(III), $\Delta: \mathrm{Fe}(\mathrm{III}))$ on $0.5 \mathrm{ppm} \mathrm{Sc}$ (III) solution under coexistence with $\mathrm{Fe}(\mathrm{III})$ at original $\mathrm{pH}$.

5) K. Saito, M. Ito, H. Yamagishi, S. Furusaki, T. Sugo and J. Okamoto, Ind. Eng. Chem. Res., 28, 1808 (1989).

6) Y. Ueki, N. C. Dafader, H. Hoshina, N. Seko and M. Tamada, Radiat. Phys. Chem., 81, 890 (2012).

7) F. Basuki, N. Seko, M. Tamada, T. Sugo and T. Kume, J. Ion Exchange, 14 (Suppl.) 209 (2003).

8) N. Seko, N. T. Y. Ninh and M. Tamada, Radiat. Phys. Chem., 79, 22 (2010). 